\title{
Meta
}

Journal des traducteurs

Translators' Journal

\section{Poirier, Éric (1995) : Le bestiaire. Dictionnaire bilingue et analogique des expressions animalières de la langue anglaise, Brossard (Québec), Linguatech}

\section{Henri Béjoint}

Volume 41, numéro 4, décembre 1996

La dénomination

URI : https://id.erudit.org/iderudit/017918ar

DOI : https://doi.org/10.7202/017918ar

Aller au sommaire du numéro

Éditeur(s)

Les Presses de l'Université de Montréal

ISSN

0026-0452 (imprimé)

1492-1421 (numérique)

Découvrir la revue

Citer ce compte rendu

Béjoint, H. (1996). Compte rendu de [Poirier, Éric (1995) : Le bestiaire. Dictionnaire bilingue et analogique des expressions animalières de la langue anglaise, Brossard (Québec), Linguatech]. Meta, 41(4), 622-631.

https://doi.org/10.7202/017918ar d'utilisation que vous pouvez consulter en ligne. 
Les traducteurs des XVIre et XVIII ${ }^{e}$ siècles connaissent évidemment la tâche ardue qu'entraîne la traduction du style de l'original. Il existe à l'endroit de ce problème trois méthodes de «solutions». La première préconise la transposition en langue-cible des procédés propres à l'auteur de la langue de départ. La deuxième défend l'idée que l'affaiblissement de l'effet de l'original est compensé par le respect de l'intégralité des idées. La troisième cherche dans la reconstitution de l'effet de l'original le véritable principe de la traduction.

Le livre de Daniel Mercier fourmille d'exemples éloquemment parlants des problèmes et des paradoxes auxquels les traducteurs de l'âge classique se heurtaient. Il a le grand mérite de présenter en une synthèse bien structurée l'idéologie traductologique d'une époque importante de l'histoire de l'humanité. L'agréable style, qui se caractérise surtout par la concision lexicale, ajoute fortement au plaisir de lecture que ce livre procure à ses lecteurs. Nous devons féliciter Daniel Mercier du précieux apport qu'il livre avec son ouvrage à l'histoire de la traduction: une histoire qui se révèle non seulement intéressante pour son contenu proprement biographique, mais aussi pour les leçons qu'elle permet de tirer du passé pour quiconque ne veut pas voir en la traduction un métier à jamais figé dans des acquis méthodologiques inébranlables. Certains livres dans le domaine de la traductologie sont, quoi qu'en dise la quatrième de couverture, «contournables». Le livre de Daniel Mercier ne l'est certainement pas: il est impératif pour tous ceux pour qui la réflexion sur les méthodologies est un moyen d'enrichir la pratique traduisante.

PAUL WIJNANDS École Supérieure de Maastricht, Maastricht, Pays-Bas

POIRIER, Éric (1995): Le bestiaire. Dictionnaire bilingue et analogique des expressions animalières de la langue anglaise, Brossard (Québec), Linguatech.

Le dictionnaire d'Éric Poirier, destiné aux traducteurs de l'anglais vers le français, contient «environ 300 expressions animalières et 900 variantes et synonymes. Il en propose à peu près 1200 équivalents». Ce qu'entend l'auteur par «expression animalière» n'est pas défini de manière très précise - il se contente de dire qu'il s'agit d'une expression «qui correspond à un nom d'animal» —, mais on comprend vite ce dont il s'agit en feuilletant le livre. Ainsi, au hasard: know a hawk from a handsaw, watch (sb / sth) like a hawk, be as prickly as a hedgehog, be as mad as a wet hen, be as scarce as hen's teeth, be like a hen with one chicken, go the whole hog, be as mad as a hornet, back the right horse, etc.

Chaque entrée est repérée par le nom de l'animal qui est utilisé dans l'expression vedette. Si le même nom d'animal est utilisé dans plusieurs expressions, les différents articles sont notés par des numéros : ainsi, know a hawk from a handsaw est HAWK, 1, et watch (sb / sth) like a hawk est HAWK, 2, etc.

La microstructure des articles se compose:

$1^{\circ}$ des variantes et synonymes anglais de l'expression entrée (trois en moyenne par entrée), que ceux-ci soient également animaliers ou pas: dans l'article HORNET, 1 , on trouve, après l'expression principale be as mad as a hornet, les deux expressions suivantes: be as mad as a wet hen, be as mad as a hatter. 
$2^{\circ}$ d'une définition, en français, de ces expressions. Dans l'entrée citée ci-dessus, la définition est la suivante : «Être dans une grande colère.»

$3^{\circ}$ d'équivalents français possibles (quatre en moyenne par entrée). Ici, «se fâcher tout rouge, être en furie, être dans une colère noire, être enragé noir (QC, FAM), être en (beau) joualvert (QC, POP), écumer comme un verrat (POP). L'auteur nous dit que ces équivalents sont classés "par ordre de préférence». On aura remarqué que «des indications sur l'origine géographique, le niveau de langue et les limites d'usage [...] accompagnent les expressions».

$4^{\circ}$ de remarques explicatives s'il y a lieu. Ainsi, l'article HORSE, 1 (back the right horse) se termine par «Dans un sens plus abstrait, on peut également dire: se mettre $d u$ côté du manche».

L'originalité de l'ouvrage réside principalement dans le premier point, les variantes en langue source, et accessoirement dans le troisième, les équivalences multiples (rares dans les dictionnaires d'expressions idiomatiques).

L'ouvrage est agrémenté de deux bibliographies — une terminologique et une générale - et de deux index, un anglais et un français, destinés à permettre «de trouver facilement une expression qui ne contient pas de nom d'animal ou, ce qui est utile aux anglophones, une expression française».

Ce dictionnaire se feuillette avec plaisir. Plaisir de voir défiler les noms d'animaux : il y en a 90, de ant à worm. Plaisir de découvrir la richesse de cette inspiration pour la langue anglaise: l'animal le plus fécond du point de vue linguistique, si l'on en croit le dictionnaire, est $d o g$ avec 32 articles, suivi de cat avec 27, horse avec 25, bird avec 21 , etc.; pig, curieusement, n'en donne que 11. Plaisir enfin de voir se suivre les utilisations, basées en particulier sur la connotation, de ces noms d'animaux, dont la plupart, il faut bien le dire, sont bien connues : l'âne est stupide, le castor industrieux, le mouton docile, etc.

Mais ce «Bestiaire» peut également être utile aux traducteurs, qui y trouveront différentes idées de traduction pour chacune des expressions retenues. On pourra trouver la nomenclature bien limitée, mais elle a au moins le mérite d'être clairement définie. Parmi les reproches qu'on peut faire, citons :

- La séparation entre les différents sens est parfois peu convaincante : par exemple, back the right horse et back the wrong horse font l'objet de deux articles séparés, alors que les deux expressions ne diffèrent que par l'adjectif. De même, BULL, 5 (hit the bull's eye dans le sens de mettre dans le mille, tirer dans le mille, faire mouche), BULL, 6 (hit the bull's eye dans le sens de faire mouche et viser juste) et BULL, 7 (hit the bull's eye dans le sens de voir juste, viser juste, mettre dans le mille, faire mouche) auraient sans doute gagné à être regroupés.

- Les équivalences françaises ne sont pas toujours fiables. Par exemple, être bête comme ses pieds et être aimable comme une porte de prison sont tous deux présentés comme équivalents de be as sour as a crab, se mettre le doigt dans l' 'eil et se mettre dans le pétrin de cook one's goose, etc. L'ouvrage devra être utilisé avec circonspection par les francophones hors du Canada. Comme on l'a vu plus haut, les québécismes sont en général indiqués, mais il subsiste des expressions dont on est en droit de se demander dans quelle aire géographique elles sont utilisées: où dit-on écumer comme un verrat, recevoir des pommes cuites (traduction de get the (big) bird from sb), être fou à triple étage (be as crazy as a loon), être un corbeau (be like a vulture), ramasser ses pénates (get one's ducks in a row), voir des rats bleus (see pink elephants), etc.? Tout cela demanderait des explications supplémentaires, certes bien difficiles à fournir dans le cadre d'un si petit ouvrage.

Ce dictionnaire monodirectionnel pourra se révéler utile au traducteur qui se trouve confronté à une difficulté particulière. Il pourra aussi servir de modèle méthodologique à 
d'autres ouvrages plus généraux qui lui emprunteraient l'idée des variantes de langue source et des équivalences multiples.

HENRI BÉJOINT

CRTT, Université Lumière, Lyon, France

- KUČERA, Antonin, CLAS, André et Jean BAUDOT (1996): Dictionnaire compact des Sciences et de la Technique. Compact Wörterbuch der exakten Naturwissenschaften und der Technik, volume 1, Français-allemand, Wiesbaden, Brandstetter Verlag, $\mathrm{XX}+1103 \mathrm{p}$.

Faire un dictionnaire scientifique et technique général est une tâche ingrate; comment en un nombre limité de pages faire le tour des millions de termes qui existent dans la quasi-infinité de domaines et sous-domaines d'une expérience humaine en constante expansion? On pourra toujours objecter qu'il en manque! Et pourtant, ce que recherchent la plupart des utilisateurs, même les traducteurs professionnels, c'est la solution à leur problème de traduction nichée entre les couvertures d'un seul volume. C'est à ce défi que le triumvirat transatlantique du Compact a décidé de répondre en produisant un dictionnaire technique général français-allemand.

Ce nouveau dictionnaire part d'une bonne base, car le Kučera anglais-allemand était déjà une référence en lexicographie spécialisée bilingue, et le volume II allemandfrançais, paru en 1991, a déjà profité de l'expérience de son aîné. En plus, le premier volume, qui paraît un peu curieusement cinq ans après le second, n'est pas un simple retournement de la paire des langues, mais comporte une mise à jour et des ajouts considérables, soit 21000 mots nouveaux, selon les auteurs, sur les quelque 94000 termes inclus.

Comment alors apprécier un dictionnaire qui couvre des domaines qui vont de l'acoustique à la zymologie, et qui sont aussi différents que la cinématographie et la physique des plasmas? La réponse est nécessairement aléatoire, mais afin d'éviter au maximum l'arbitraire, nous avons essayé de définir une tactique de sondage. Nous avons choisi de soumettre le nouveau Kučera à une épreuve inégale, à savoir une comparaison de ce dictionnaire et de quelques dictionnaires spécialisés monothématiques. On peut s'attendre à ce que ces derniers atteignent une profondeur de détail qu'un dictionnaire généraliste ne vise pas, mais on peut espérer en même temps que celui-ci comporte les termes les plus essentiels et les plus productifs.

Le sondage fait ainsi appel à quelques dictionnaires spécialisés français-allemand (avec éventuellement d'autres langues), dans le but d'évaluer le degré de complétude du Compact et de signaler, en passant, quelques-unes de ses techniques de présentation. Cette comparaison est facilitée par l'indication systématique du domaine pour chaque mot du dictionnaire, ce qui par ailleurs représente une précision très utile absente de beaucoup de dictionnaires techniques généraux.

On peut supposer que ce dictionnaire privilégie expressément les techniques dures, car il ne fait pas mention de domaines plus «mous» comme ceux de la banque ou des assurances: ni assurances ni banque, par exemple, ne figurent parmi les abréviations de 